PROCEEDINGS OF THE

AMERICAN MATHEMATICAL SOCIETY

Volume 134, Number 11, November 2006, Pages 3115-3121

S 0002-9939(06)08428-0

Article electronically published on May 12, 2006

\title{
THE EXISTENCE OF FINITELY GENERATED MODULES OF FINITE GORENSTEIN INJECTIVE DIMENSION
}

\author{
RYO TAKAHASHI \\ (Communicated by Bernd Ulrich)
}

\begin{abstract}
In this note, we study commutative Noetherian local rings having finitely generated modules of finite Gorenstein injective dimension. In particular, we consider whether such rings are Cohen-Macaulay.
\end{abstract}

\section{INTRODUCTION}

Throughout this note, we assume that all rings are commutative and Noetherian. The following celebrated theorems, where the second one has been called Bass' conjecture, are obtained by virtue of the (Peskine-Szpiro) intersection theorem. For the details, see [4, Corollaries 9.4.6 and 9.6.2].

Theorem A. A local ring is Cohen-Macaulay if it admits a nonzero CohenMacaulay module of finite projective dimension.

Theorem B. A local ring is Cohen-Macaulay if it admits a nonzero finitely generated module of finite injective dimension.

In the sixties, Auslander 1 introduced Gorenstein dimension (abbr. G-dimension) as a homological invariant for finitely generated modules, and developed its notion with Bridger [2. This invariant is an analogue of projective dimension; any finitely generated module of finite projective dimension has finite G-dimension.

Several decades later, Gorenstein projective dimension (abbr. G-projective dimension) was defined as an extension of G-dimension to modules that are not necessarily finitely generated, and Gorenstein injective dimension (abbr. G-injective dimension) was defined as a dual version of G-projective dimension. The notions of these dimensions are based on the work of Enochs and Jenda 7]. G-injective dimension is a refinement of the usual injective dimension in the sense that any module of finite injective dimension has finite G-injective dimension. So far these dimensions have been extended to complexes of modules.

Now, it is natural to ask the following questions, which are generalizations of Theorem $\mathrm{A}$ and Theorem $\mathrm{B}$, respectively.

Received by the editors August 12, 2004 and, in revised form, October 1, 2004, January 31, 2005, and May 31, 2005.

2000 Mathematics Subject Classification. Primary 13D05; Secondary 13 H10.

Key words and phrases. G-injective dimension (Gorenstein injective dimension), G-dimension (Gorenstein dimension). 
Question A. Is a local ring Cohen-Macaulay if it admits a nonzero CohenMacaulay module of finite G-dimension?

Question B. Is a local ring Cohen-Macaulay if it admits a nonzero finitely generated module of finite G-injective dimension?

Both of these questions are presented in Christensen's book [5], while the second one is also found in 9 .

The author proved that Question A has an affirmative answer for any local ring of type one:

Theorem 1.1 ([11, Theorem 2.3]). The following are equivalent for a local ring $R$ :

(1) $R$ is Gorenstein;

(2) $R$ admits an ideal I of finite $G$-dimension such that $R / I$ is Gorenstein;

(3) $R$ admits a nonzero Cohen-Macaulay module of type one and of finite $G$ dimension;

(4) $R$ has type one and admits a nonzero Cohen-Macaulay module of finite G-dimension.

In this note, we will consider Question B. The main theorems of this note are the following two theorems.

Theorem 3.4. Let $R$ be a local ring with a dualizing complex. Then for a nonzero finitely generated $R$-module $M$, there is an inequality

$$
\operatorname{dim} M \leq \operatorname{Gid} M .
$$

Theorem 3.5. Let $R$ be a local ring with a dualizing complex, and let $M$ be a nonzero finitely generated $R$-module of finite $G$-injective dimension.

(1) If $\operatorname{dim} M=\operatorname{dim} R$, then $R$ is Cohen-Macaulay.

(2) (i) If $M$ is cyclic, then $R$ has type one.

(ii) Suppose that $R$ has type one. If $M$ is Cohen-Macaulay or $\operatorname{depth} M \geq$ $\operatorname{depth} R$, then $R$ is Gorenstein.

In the next section we will prepare some definitions and lemmas, and in the third (final) section we will state and prove our results.

\section{Preliminaries}

In this section, we will write only results to be used later. Throughout this section, let $R$ be a local ring. In the following, an $R$-complex will mean a chain complex $X=\left(\cdots \rightarrow X_{i+1} \rightarrow X_{i} \rightarrow X_{i-1} \rightarrow \cdots\right)$ of $R$-modules, and a bounded $R$-complex will mean a homologically bounded $R$-complex, namely an $R$-complex $X$ such that $\mathrm{H}_{i}(X)=0$ for any $i \gg 0$ and $i \ll 0$. An isomorphism of $R$-complexes will mean an isomorphism in the derived category of the category of $R$-modules.

Definition 2.1 ([5, (2.3.2)]).

(1) We denote by $\mathcal{G}(R)$ the class of all finitely generated $R$-modules $M$ satisfying the following three conditions:

(i) The natural homomorphism $M \rightarrow \operatorname{Hom}_{R}\left(\operatorname{Hom}_{R}(M, R), R\right)$ is an isomorphism;

(ii) $\operatorname{Ext}_{R}^{i}(M, R)=0$ for any $i>0$;

(iii) $\operatorname{Ext}_{R}^{i}\left(\operatorname{Hom}_{R}(M, R), R\right)=0$ for any $i>0$. 
(2) Let $X$ be an $R$-complex and $n$ an integer. If there exists an $R$-complex of the form

$$
A=\left(0 \rightarrow A_{n} \rightarrow A_{n-1} \rightarrow \cdots \rightarrow A_{m+1} \rightarrow A_{m} \rightarrow 0\right),
$$

which is isomorphic to $X$ such that $A_{i} \in \mathcal{G}(R)$ for any $i$, then we say that $X$ has $G$-dimension at most $n$ and write $\operatorname{Gdim}_{R} X \leq n$.

Let $X$ be an $R$-complex. If $X \cong 0$, i.e. $X$ is exact, then we set $\operatorname{Gdim}_{R} X=-\infty$. If $\operatorname{Gdim}_{R} X \leq n$ for some integer $n$, then we say that $X$ has finite G-dimension and write $\operatorname{Gdim}_{R} X<\infty$. If $\operatorname{Gdim}_{R} X \not \leq n$ for any integer $n$, then we say that $X$ has infinite G-dimension and write $\operatorname{Gdim}_{R} X=\infty$. If $\operatorname{Gdim}_{R} X \leq n$ but $\operatorname{Gdim}_{R} X \not \leq$ $n-1$, then we say that $X$ has G-dimension $n$ and write $\operatorname{Gdim}_{R} X=n$.

Lemma 2.2 ([5, (2.3.8), (2.3.13)], [3, 8.7]).

(1) The following are equivalent for a bounded $R$-complex $X$ with finitely generated homology:

(i) $\operatorname{Gdim}_{R} X<\infty$.

(ii) The complex $\mathbf{R} \operatorname{Hom}_{R}(X, R)$ is bounded, and the natural homomorphism $X \rightarrow \mathbf{R H o m}_{R}\left(\mathbf{R H o m}_{R}(X, R), R\right)$ is an isomorphism.

(2) Let $X$ be a bounded $R$-complex with finitely generated homology. If $\operatorname{Gdim}_{R} X$ $<\infty$, then

$$
\operatorname{Gdim}_{R} X=-\inf \mathbf{R} \operatorname{Hom}_{R}(X, R)=\operatorname{depth} R-\operatorname{depth}_{R} X .
$$

(3) Let $M$ be a finitely generated $R$-module.

(i) For an $M$-regular element $x \in R$,

$$
\operatorname{Gdim}_{R} M / x M=\operatorname{Gdim}_{R} M+1 .
$$

(ii) Let $R \rightarrow S$ be a faithfully flat homomorphism of local rings. Then

$$
\operatorname{Gdim}_{S} M \otimes_{R} S=\operatorname{Gdim}_{R} M .
$$

Definition 2.3 ([5, (6.1.1), (6.2.2)]).

(1) An $R$-module $M$ is said to be $G$-injective if there exists an exact complex

$$
C=\left(\cdots \stackrel{d_{3}}{\longrightarrow} C_{2} \stackrel{d_{2}}{\longrightarrow} C_{1} \stackrel{d_{1}}{\longrightarrow} C_{0} \stackrel{d_{0}}{\longrightarrow} C_{-1} \stackrel{d_{-1}}{\longrightarrow} \cdots\right)
$$

of injective $R$-modules with $M \cong \operatorname{Ker}\left(d_{0}\right)$ such that the $\operatorname{complex} \operatorname{Hom}_{R}(T, C)$ is also exact for any injective $R$-module $T$.

(2) Let $X$ be an $R$-complex and $n$ an integer. If there exists an $R$-complex of the form

$$
A=\left(0 \rightarrow A_{m} \rightarrow A_{m-1} \rightarrow \cdots \rightarrow A_{-n+1} \rightarrow A_{-n} \rightarrow 0\right),
$$

which is isomorphic to $X$ such that $A_{i}$ is G-injective for any $i$, then we say that $X$ has $G$-injective dimension at most $n$ and write $\operatorname{Gid}_{R} X \leq n$.

The conditions $\operatorname{Gid}_{R} X=-\infty, \operatorname{Gid}_{R} X<\infty, \operatorname{Gid}_{R} X=\infty, \operatorname{and}_{\operatorname{Gid}_{R}} X=n$ are defined similarly to G-dimension.

Lemma 2.4 ([5, (6.2.3)], [6, (2.12), (6.3), (6.4)]).

(1) For a bounded $R$-complex $X$,

$$
\operatorname{Gid}_{R} X \leq \operatorname{id}_{R} X .
$$


(2) Assume that $R$ admits a dualizing complex $D$.

(i) For a bounded R-complex $X$ with finitely generated homology,

$\operatorname{Gid}_{R} X<\infty \Leftrightarrow \operatorname{Gdim}_{R} \mathbf{R} \operatorname{Hom}_{R}(X, D)<\infty$.

(ii) Let $M$ be a nonzero finitely generated $R$-module of finite $G$-injective dimension. Then

$$
\operatorname{Gid}_{R} M=\operatorname{depth} R .
$$

\section{MAIN THEOREMS}

First of all, we remark that Theorem $\mathrm{B}$ implies Theorem $\mathrm{A}$. Indeed, let $R$ be a local ring and $M$ a Cohen-Macaulay $R$-module of finite projective dimension. Setting depth $M=\operatorname{dim} M=n$, we have an $M$-sequence $\boldsymbol{x}=x_{1}, x_{2}, \ldots, x_{n}$ in $R$ such that $M / \boldsymbol{x} M$ has finite length. Then since the $R$-module $M / \boldsymbol{x} M$ also has finite projective dimension, the dual $R$-module $(M / \boldsymbol{x} M)^{\vee}$ has finite length and finite injective dimension, where $(-)^{\vee}$ denotes the Matlis dual over $R$. Hence it follows from Theorem $\mathrm{B}$ that $R$ is Cohen-Macaulay. Thus Theorem B implies Theorem A,

Similarly, we have the following.

Proposition 3.1. If Question B has an affirmative answer, then so has Question A.

Proof. Let $R$ be a local ring and $M$ a Cohen-Macaulay $R$-module of finite Gdimension. We want to show that $R$ is Cohen-Macaulay under the assumption that Question $\mathrm{B}$ has an affirmative answer. Replacing $R$ and $M$ by their completions respectively, we may assume that $R$ is complete; see Lemma 2.2(3)(ii). Since $M$ is a Cohen-Macaulay $R$-module, there exists an $M$-sequence $\boldsymbol{x}$ in $R$ such that $M / \boldsymbol{x} M$ has finite length. Lemma 2.2(3)(i) says that the $R$-module $M / \boldsymbol{x} M$ has finite Gdimension. Replacing $M$ by $M / \boldsymbol{x} M$, we may assume that $M$ has finite length and finite G-dimension. Note that $\mathbf{R} \operatorname{Hom}_{R}(M, D)$ is isomorphic to $M^{\vee}$ up to shift, where $D$ is a dualizing complex of $R$. Hence, according to Lemma 2.4(2)(i), $M^{\vee}$ has finite length and finite G-injective dimension. Since we are assuming that Question $\mathrm{B}$ has an affirmative answer, we conclude that $R$ is Cohen-Macaulay, as desired.

From now on, we will study Question B over a local ring admitting a dualizing complex, and prove the main theorems of this note.

Let $(R, \mathfrak{m}, k)$ be a local ring. We set $d=\operatorname{dim} R$ and $t=\operatorname{depth} R$. Let $D=$ $\left(0 \rightarrow D_{0} \rightarrow D_{-1} \rightarrow \cdots \rightarrow D_{-d} \rightarrow 0\right)$ be a dualizing complex of $R$, where $D_{i}=$ $\bigoplus_{\mathfrak{p} \in \operatorname{Spec} R, \operatorname{dim} R / \mathfrak{p}=d+i} E_{R}(R / \mathfrak{p})$. The local duality theorem yields inf $D=t-d$.

Let $M \neq 0$ be a finitely generated $R$-module with Gid $M<\infty$. Put $X=$ $\mathbf{R H o m}_{R}(M, D)$. Lemma 2.4 (2)(i) implies $\operatorname{Gdim} X<\infty$. Since depth $X=\operatorname{depth} D$ $=d$ by [5, (A.6.4)], we have inf $\mathbf{R H o m}_{R}(X, R)=d-t$ by Lemma 2.2(2). Since $\mathbf{R H o m}_{R}(X, R) \cong \mathbf{R} \operatorname{Hom}_{R}(D, M)$, we have sup $\mathbf{R H o m}_{R}(X, R)=\sup \mathbf{R} \operatorname{Hom}_{R}(D, M)$ $\leq d-t$ by [5. (A.4.6.1)]. Hence $\sup \mathbf{R} \operatorname{Hom}_{R}(X, R)=\inf \mathbf{R} \operatorname{Hom}_{R}(X, R)=d-t$. Setting $N=\mathrm{H}_{d-t}\left(\mathbf{R H o m}_{R}(X, R)\right)$, we get $\mathbf{R H o m}_{R}(X, R) \cong N[d-t]$. Lemma 2.2(1) implies

$$
\operatorname{Gdim} N<\infty \text {. }
$$

Noting that $N \cong \mathrm{H}_{d-t}\left(\mathbf{R} \operatorname{Hom}_{R}(D, M)\right)$ and $\inf D=t-d$, we obtain

$$
N \cong \operatorname{Hom}_{R}\left(\mathrm{H}_{t-d}(D), M\right) \text {. }
$$


Remark 3.2. In the above part, we constructed the complex $\mathbf{R H o m}_{R}(D, M)$ of finite G-dimension from a module $M$ of finite G-injective dimension. A deeper investigation in connection with this fact has been made by Foxby; he has given an equivalence between the Auslander class and the Bass class, which are certain full subcategories of the category of complexes. This equivalence is said to be Foxby equivalence. For the definition and the properties, see [5, Section 3.3].

Lemma 3.3. With the notation introduced above,

$$
\operatorname{depth} M=\operatorname{depth} N \leq \operatorname{dim} N \leq \operatorname{dim} M \leq t .
$$

Proof. By [5, (A.8.5.1), (A.8.5.2)] we get inf $X=\operatorname{depth} M-d$ and $\sup X=\operatorname{dim} M-$ $d$. Note that $\operatorname{RHom}_{R}(N, R) \cong X[d-t]$. By (3.1) and Lemma 2.2(2) we obtain $t-\operatorname{depth} N=\operatorname{Gdim} N=-\inf \mathbf{R} \operatorname{Hom}_{R}(N, R)=-\inf X-(d-t)=t-\operatorname{depth} M$, and $0 \leq \operatorname{grade} N=-\sup \mathbf{R} \operatorname{Hom}_{R}(N, R)=-\sup X-(d-t)=t-\operatorname{dim} M$. Hence $\operatorname{depth} N=\operatorname{depth} M$ and $\operatorname{dim} M \leq t$. On the other hand, since Supp $N \subseteq \operatorname{Supp} M$ by (3.2), we have $\operatorname{dim} N \leq \operatorname{dim} M$. Thus the proof of the lemma is completed.

Let $R$ be a local ring and $M$ a nonzero finitely generated $R$-module. Then it is well known that there is an inequality

$$
\operatorname{dim} M \leq \operatorname{id} M .
$$

(For the proof, see [4, Theorem 3.1.17].) Combining Lemma 3.3 with Lemma 2.4(2)(ii) extends this formula of injective dimension to that of G-injective dimension.

Theorem 3.4. Let $R$ be a local ring with a dualizing complex. Then for a nonzero finitely generated $R$-module $M$, there is an inequality

$$
\operatorname{dim} M \leq \operatorname{Gid} M .
$$

Recall that the type of a finitely generated module $M$ over a local ring $R$ with residue field $k$ is defined to be the dimension of the $k$-vector space $\operatorname{Ext}_{R}^{t}(k, M)$ where $t=\operatorname{depth}_{R} M$. Using Lemma 3.3 again, we can give a result with relation to Question B in the first section of this note.

Theorem 3.5. Let $R$ be a local ring with a dualizing complex, and let $M$ be a nonzero finitely generated $R$-module of finite $G$-injective dimension.

(1) If $\operatorname{dim} M=\operatorname{dim} R$, then $R$ is Cohen-Macaulay.

(2) (i) If $M$ is cyclic, then $R$ has type one.

(ii) Suppose that $R$ has type one. If $M$ is Cohen-Macaulay or $\operatorname{depth} M \geq$ depth $R$, then $R$ is Gorenstein.

Proof. (1) This assertion immediately follows from Lemma 3.3

(2) We use the same notation as in the first part of this section.

(i) Set $Y=\mathbf{R H o m}_{R}(X, R)$. Using Lemma 2.2(1), we get isomorphisms

$$
\mathbf{R H o m}_{R}(M, k)[-d] \cong \mathbf{R H o m}_{R}(k, X) \cong \mathbf{R H o m}_{R}\left(Y, \mathbf{R H o m}_{R}(k, R)\right) .
$$

Since $M$ is cyclic, comparing the $(-d)$ th homology modules, we obtain $k \cong$ $\operatorname{Ext}_{R}^{t}(k, R)$ (cf. [5. (A.4.6)]). This says that Rhas type one.

(ii) It follows from Lemma 3.3 and (3.1) that $N$ is a Cohen-Macaulay $R$-module of finite G-dimension. Since $R$ has type one, Theorem 1.1 implies that $R$ is Gorenstein. 
Corollary 3.6. Let $R$ be a local ring of type one with a dualizing complex. If there is a finitely generated $G$-injective $R$-module, then $R$ is Artinian Gorenstein.

Proof. Let $M$ be a finitely generated G-injective $R$-module. Since Gid $M=0$, we have depth $R=0$ by Lemma 2.4(2)(ii). Therefore Theorem 3.5(2)(ii) implies that $R$ is Gorenstein, and $\operatorname{dim} R=\operatorname{depth} R=0$.

Holm [8, Theorem 2.1] proved that any ring of finite G-injective dimension is Gorenstein. Letting $M=R$ in Theorem 3.5(2), we get his theorem for a local ring with a dualizing complex.

Corollary 3.7 (Holm). Let $R$ be a local ring with a dualizing complex. If $\operatorname{Gid} R<$ $\infty$, then $R$ is Gorenstein.

Peskine and Szpiro [10. Chapitre II, Théorème (5.5)] proved that a local ring is Gorenstein if it has a nonzero cyclic module of finite injective dimension. Taking Lemma 2.4(1) and the above results into account, we end this note by stating a conjecture.

Conjecture 3.8. Let $R$ be a local ring. If there exists a nonzero cyclic $R$-module $M$ of finite G-injective dimension, then $R$ is Gorenstein.

\section{ACKNOWLEDGMENTS}

The author would like to thank an anonymous referee for careful reading and helpful comments.

\section{REFERENCES}

[1] M. Anneaux, de Gorenstein, et torsion en algèbre commutative, Séminaire d'Algèbre Commutative dirigé par Pierre Samuel, 1966/67. Texte rédigé, d'après des exposés de Maurice Auslander, Marquerite Mangeney, Christian Peskine et Lucien Szpiro. École Normale Supérieure de Jeunes Filles, Secrétariat mathématique, Paris 1967. MR0225844 (37:1435)

[2] M. Auslander, M. Bridger, Stable module theory, Memoirs of the American Mathematical Society, No. 94, American Mathematical Society, Providence, R.I., 1969. MR0269685 (42:4580)

[3] L. L. Avramov, Homological dimensions and related invariants of modules over local rings, Representations of algebra. Vol. I, II, 1-39, Beijing Norm. Univ. Press, Beijing, 2002. MR2067368 (2005d:13022)

[4] W. Bruns, J. Herzog, Cohen-Macaulay rings, Revised edition. Cambridge Studies in Advanced Mathematics, 39, Cambridge University Press, Cambridge, 1998. MR.1251956 (95h:13020)

[5] L. W. Christensen, Gorenstein dimensions, Lecture Notes in Mathematics, 1747, SpringerVerlag, Berlin, 2000. MR $1799866(2002 \mathrm{e}: 13032)$

[6] L. W. A. Christensen, A. Frankild, H. Holm, On Gorenstein projective, injective and flat dimensions-a functorial description with applications, preprint (arXiv:math.AC/0403156 v3 17 Mar 2004).

[7] E. E. Enochs, O. M. G. Jenda, Gorenstein injective and projective modules, Math. Z. 220 (1995), no. 4, 611-633. MR1363858 (97c:16011)

[8] H. Holm, Rings with finite Gorenstein injective dimension, Proc. Amer. Math. Soc. 132 (2004), no. 5, 1279-1283. MR2053331(2005a:13031)

[9] L. Khatami, S. Yassemi, Gorenstein injective dimension, Bass formula and Gorenstein rings. preprint (arXiv:math.AC/03125132 1 Jun 2004). 
[10] C. Peskine, L. Szpiro, Dimension projective finie et cohomologie locale. Applications a la demonstration de conjectures de M. Auslander, H. Bass et A. Grothendieck. Inst. Hautes Etudes Sci. Publ. Math., No. 42, (1973), 47-119. MR0374130 (51:10330)

[11] R. Takahashi, Some characterizations of Gorenstein local rings in terms of G-dimension. Acta Math. Hungar. 104 (2004), no. 4, 315-322. MR2082781(2005f:13015)

Department of Mathematics, Faculty of Science, Okayama University, 1-1, Naka 3Chome, Tsushima, Okayama 700-8530, JaPan

E-mail address: takahasi@math.okayama-u.ac.jp

Current address: Department of Mathematics, School of Science and Technology, Meiji University, 1-1-1 Higashimita, Tama-ku, Kawasaki 214-8571, Japan

E-mail address: takahasi@math.meiji.ac.jp 